\title{
Molecular systematics of genus Bulbophyllum (Orchidaceae) in Peninsular Malaysia based on combined nuclear and plastid DNA sequences
}

\begin{abstract}
Phylogenetic relationships were inferred for representative Bulbophyllum species of 13 sections from subtribe Bulbophyllinae (Epidendroideae, Orchidaceae) in Peninsular Malaysia. The combined data matrix consists of sequences from ITS nuclear gene region and trnL-F, matK, and rbcL plastid gene regions with 3114 characters. Molecular data were analysed using parsimony and Bayesian inference. The results show that several recognized sections are monophyletic. Section Hirtula with paraphyletic status must split up and section Desmosanthes contain misplaced elements. Furthermore, generic status of Cirrhopetalum and Epicrianthes cannot be supported, because they are deeply embedded within the genus Bulbophyllum. Section Desmosanthes is recognized as the closest group to section Cirrhopetalum; therefore, they can be merged in some aspects.
\end{abstract}

Keyword: Bulbophyllum; Monophyly; Orchidaceae; Peninsular Malaysia; Phylogenetic 